Revista de Investigación

Académica sin Frontera

ISSN: 2007-8870

\title{
http://revistainvestigacionacademicasinfrontera.com
}

Recibido el 12 de octubre de 2019. Dictaminado mediante arbitraje favorablemente 20 de diciembre de 2019.

\section{El marketing en la estrategia de gestión de las pymes}

\author{
María Lizett Zolano Sánchez ${ }^{1}$ \\ Universidad de Sonora, Unidad Norte. \\ Martín Guillermo Durán Acosta ${ }^{2}$ \\ Universidad de sonora, unidad centro. \\ María Guadalupe Torres Figueroa ${ }^{3}$ \\ Universidad de Sonora, unidad norte
}

\begin{abstract}
Resumen
Este trabajo de investigación se lleva a cabo bajo un enfoque metodológico transversal, donde se utilizó una entrevista respaldada por un cuestionario estructurado que toma como referencia a las Pymes de las ciudades de Nogales, Santa Ana, Caborca y San Luís Río Colorado ubicadas en la zona norte del estado de Sonora, con una fuerza laboral entre 10 y 250 trabajadores y una antigüedad mínima de más de cinco años en el mercado. El objetivo es determinar, con base en las opiniones de los gerentes, cual es el trabajo de marketing que utilizan en la gestión de sus negocios, lo que ayuda a generar estrategias dirigidas a la competitividad empresarial. Los principales resultados muestran que, de acuerdo con las opiniones de los gerentes, son conscientes de la importancia de la competencia en el mercado, pero aparentemente no saben cómo crear estrategias para enfrentarla. Evidentemente no dan valor al marketing como una herramienta para lograr competitividad empresarial, y gestionan sus negocios de la manera más tradicional, en función de su experiencia y en muchos casos por intuición, sin darse cuenta que están haciendo marketing.

Palabras Claves: Marketing, Estrategia, Pymes, Competitividad Empresarial.
\end{abstract}

\footnotetext{
${ }^{1}$ Departamento de Ciencias Administrativas y Agropecuarias, Universidad de Sonora, Unidad Norte. Carretera Internacional y Av. 16 de septiembre, Santa Ana, Sonora, México Tel. 01(641)3241120 correo electrónico: lizoza@ hotmail.com

2 Departamento de Contabilidad, Universidad de Sonora, Unidad Centro. Blvd. Luis Encinas y Rosales s/n, Colonia Centro, Hermosillo, Sonora, México tel. 01(662)2592168 correo electrónico: martin.duran@unison.mx

${ }^{3}$ Departamento de Ciencias Económico Administrativas, Universidad de Sonora, Unidad Norte. Avenida Universidad s/n, Nogales, Sonora, México Tel 01(631)3207734 correo electrónico: Guadalupe.torres@ unison.mx
} 
Revista de Investigación

Académica sin Frontera

Año 12.

Núm. 31

ISSN: 2007-8870

\title{
http://revistainvestigacionacademicasinfrontera.com
}

Recibido el 12 de octubre de 2019. Dictaminado mediante arbitraje favorablemente 20 de diciembre de 2019.

\begin{abstract}
This research work is carried out under a cross-sectional methodological approach, where an interview was used supported by a structured questionnaire taking as reference the SMEs of the cities of Nogales, Santa Ana, Caborca and San Luis Río Colorado located in the zone northern of the state of Sonora, with a workforce between 10 and 250 workers and a minimum age of more than five years in the market. The objective is to determine, based on the opinions of the managers, which is the marketing work that they use in the management of their businesses, which helps to generate strategies aimed at business competitiveness. The main results show that, according to managers' opinions, they are aware of the importance of market competition, but apparently they do not know how to create strategies to deal with it. They do not really give marketing value as a tool to achieve business competitiveness, and they manage their businesses in the most traditional way, based on their experience and in many cases by intuition, without realizing that they are doing marketing.
\end{abstract}

Keywords: Marketing, Strategy, SMEs, Business Competitiveness.

\section{Introducción}

Hoy en día, México enfrenta un gran reto en materia de productividad. La evidencia señala que la productividad total de los factores en la economía ha decrecido durante los últimos 30 años a una tasa promedio anual de $0.7 \%$, insuficiente para sostener una economía en crecimiento (INEGI, 2018). En el caso de las empresas, la productividad de las grandes empresas modernas creció desde 1999 a una tasa de crecimiento promedio anual del 5.8\%, las empresas tradicionales disminuyeron su productividad a una tasa del $6.5 \%$ anual. Las pequeñas y medianas empresas (Pymes), quienes tienen una alta participación en la generación de la riqueza del país, cada vez más se enfrentan al gran problema de baja productividad y en los últimos 3 años no han tenido el impacto que se esperaría en nuestra economía, su desempeño productivo limita el desarrollo nacional y fomenta la desigualdad entre las empresas, sectores y regiones económicas (Paredes, 2011; INEGI, 2018). 
Revista de Investigación

Académica sin Frontera

Año 12.

Núm. 31

ISSN: 2007-8870

\section{http://revistainvestigacionacademicasinfrontera.com}

Recibido el 12 de octubre de 2019. Dictaminado mediante arbitraje favorablemente 20 de diciembre de 2019.

Uno de los más grandes retos que enfrentan las Pymes es sobresalir, pero los presupuestos son limitados y el costo de hacer campañas que en realidad aumenten las ventas parece de momentos imposible, ya que la raíz de esta situación está en la asignación de recursos, porque las Pymes más que cualquier tipo de empresa tienen que cuidar muy bien la forma en la que invierten su dinero (Góngora y Madrid, 2010). Las prácticas de marketing tradicional propuestas por muchos autores, son basadas en modelos desarrollados para las grandes organizaciones (Coviello, Brodie y Munro, 2000; Hills et al., 2008). Sin embargo, por la estructura, condiciones y necesidades de las Pymes, el marketing en estas empresas difiere de las prácticas realizadas en las grandes organizaciones, o el marketing presentado en los libros de texto. (Carson y Gilmore, 2000; Stokes, 2000; Coviello et al., 2000; Gilmore, Carson y Grant., 2001; Zontanos y Anderson, 2004; 2006; Martin 2009; Morrish y Deacon, 2009; Reijonen, 2010).

En este sentido, las estrategias de marketing es un tema que cada vez interesa más a los gerentes de las Pymes. Por ello, el objetivo de este proyecto de investigación es determinar, con base en las opiniones de los gerentes de las Pymes, cual es el trabajo de marketing que utilizan en la gestión de sus negocios, que ayude a la generación de estrategias encaminadas a la competitividad empresarial.

\section{Antecedentes}

Existe evidencia que comprueba que ser nueva y pequeña empresa presenta dificultades especiales, como son situaciones estructurales, de gestión, económicas y sociales que se desarrollan en su entorno. Sin embargo, a pesar de afrontar estas dificultades, también tienden a ser más creativas e innovadoras que las grandes empresas (McCartan-Quinn y Carson, 2003; Gruber, 2004; Paredes, 2011). La creatividad y la innovación a través de un buen trabajo de 
"El saber de mis hijos hará mi grandeza"

Año 12. Núm. 31
Revista de Investigación

Académica sin Frontera ISSN: 2007-8870

\section{http://revistainvestigacionacademicasinfrontera.com}

Recibido el 12 de octubre de 2019. Dictaminado mediante arbitraje favorablemente 20 de diciembre de 2019.

marketing, es fundamental para el buen desempeño de la empresa (Jaworski y Kohli, 1993), esta herramienta de gestión empresarial es identificada como una de las actividades más importantes para la sobrevivencia y crecimiento de las pequeñas empresas, pero a la vez como uno de los principales problemas para los propietarios/gerentes (Stokes, 2000). Enfoques como el Entrepreneurial Marketing, que no es otra cosa que una estrategia de marketing única que se diferencia de las prácticas de marketing tradicionales, han surgido como la opción más adecuada para empresas que operan en un contexto Pyme (Hills et al., 2008; Paredes, 2011).

La función de marketing en la Pyme ha sido relacionada con ventas y promoción (O’Dwyer, Gilmore y Carson, 2009). Por su parte, Reijonen (2010), menciona que el marketing para los pequeños empresarios está relacionado con la promoción, luego con las ventas. Las personas encargadas de realizar marketing en las Pymes consideran que este es sinónimo de ventas y publicidad (Marcati, Guido y Peluso, 2008), dando importancia solamente a estos elementos

debido a que las empresas han obtenido resultados favorables sin haber realizado una planificación de las actividades de marketing (Stokes, 2000). El rezago de información de éxito de las Pymes a través del marketing, se debe a que antes el empresario sólo se preocupaba por la producción, porque gracias a la nula competencia tenía la posibilidad de vender fácilmente todo lo que producía, dando cambio a esta medida a través de una efectiva gestión empresarial (Reijonen, 2010).

En la actualidad se tiene que preocupar por el marketing, el cual tarda más en dar resultados debido a que es un proceso complejo que requiere el estudio constante del mercado, y para lo cual es fundamental contar con un plan de marketing, dado que este puede llegar a afectar las ventas, la comunicación, el pricing (políticas de precios), y en algunos casos la distribución. El marketing es fundamental en las Pymes porque estas tienen objetivos claros enfocados a calidad 
Revista de Investigación

Académica sin Frontera

Año 12.

Núm. 31
ISSN: 2007-8870

\section{http://revistainvestigacionacademicasinfrontera.com}

Recibido el 12 de octubre de 2019. Dictaminado mediante arbitraje favorablemente 20 de diciembre de 2019.

y productividad, que para su logro es necesario estar seguros de que sus esfuerzos en el año son adecuados en la consecución de estas metas (Villalobos, 2015).

Hoy en día, las empresas ya no cuentan con la posibilidad de ofertar productos que no tengan relación con lo que el mercado necesita; es indispensable que se adapten a lo que los consumidores desean, por lo que es necesario que el marketing cuente con una dimensión estratégica debido a que los mercados actuales cuentan con clientes que están más informados y son cada vez más exigentes, la existencia de una libre competencia y el rápido desarrollo tecnológico (Agius, 2015). Por su parte las Pymes están experimentando cambios, la preocupación por el negocio ha ido posicionándose en la conciencia del pequeño empresario y de allí la fuerte apuesta por este tipo de herramientas de dirección y gestión empresarial, de posicionamiento comercial, de fidelización o de planeación que brinda el marketing en ésta área, ya que incluso, puede llegar a significar la ubicación de productos fuera de las fronteras nacionales si se le emplea inteligentemente (Alarcon y Granda, 2018).

A las Pymes, les cuesta trabajo adaptarse a esos cambios y la preocupación por el negocio ha ido posicionándose en la conciencia de estos empresarios y de allí la fuerte apuesta por contar con marketing como herramientas de dirección y gestión empresarial, que permita lograr un posicionamiento comercial, establecer una estrategia de mercadeo que influya en el proceso de decisión de compra, que le impactaría principalmente en el logro de una mejor competitividad empresarial (Kotler y Amstrong; 2010; Lamb, Hair y McDaniel; 2011).

Por lo antes dicho, es importante considerar que el marketing es la actividad comprendida por un conjunto de procesos enfocados para crear, comunicar y realizar el intercambio de ofertas de productos, servicios e ideas que son de valor para los clientes, socios, empleados y la sociedad en general; es una importante herramienta que permite a las empresas ser competitivas y hacer frente a los distintos problemas que se presentan en los mercados actuales. Las Pymes consideran que 
Revista de Investigación

Académica sin Frontera

Año 12.

Núm. 31

ISSN: 2007-8870

\section{http://revistainvestigacionacademicasinfrontera.com}

Recibido el 12 de octubre de 2019. Dictaminado mediante arbitraje favorablemente 20 de diciembre de 2019.

realizar una inversión en marketing es un lujo al cual solo pueden acceder las grandes empresas, cuando en el mundo actual contar con un buen plan de marketing se ha convertido en una necesidad de empresas de todo tamaño.

\section{Objetivo}

El objetivo de esta investigación es determinar, con base en las opiniones de los gerentes de las Pymes de las ciudades de Nogales, Santa Ana, Caborca y San Luís Río Colorado ubicados en la zona norte del estado de Sonora, cual es el trabajo de marketing que utilizan en la gestión de sus negocios, que ayude a la generación de estrategias encaminadas a la competitividad empresarial.

\section{Metodología}

\section{Diseño de la investigación}

Este estudio se basa en una investigación de campo bajo un enfoque metodológico de corte transversal con el objetivo de determinar, con base en las opiniones de los gerentes de Pymes de las ciudades de Nogales, Santa Ana, Caborca y San Luís Río Colorado ubicados en la zona norte del estado de Sonora, cual es el trabajo de marketing que utilizan en la gestión de sus negocios, que ayude a la generación de estrategias encaminadas a la competitividad empresarial.

\section{Elección y tipo de muestra}

El período de trabajo de la investigación se realizó desde junio del 2018 a mayo del 2019. El universo seleccionado para esta investigación se eligió bajo el mismo criterio de estratificación de las empresas utilizado por el INEGI para los censos económicos 2017 de México que fueron obtenidas en noviembre del 2018 y acordados por los integrantes de la investigación, al cual se le anexarán otros criterios tales como: Pymes del sector industrial y comercial ubicadas en las 
Revista de Investigación

Académica sin Frontera ISSN: 2007-8870

Núm. 31

\section{http://revistainvestigacionacademicasinfrontera.com}

Recibido el 12 de octubre de 2019. Dictaminado mediante arbitraje favorablemente 20 de diciembre de 2019.

ciudades de Nogales, Santa Ana, Caborca y San Luís Río Colorado ubicados en la zona norte del estado de Sonora, México con una fuerza laboral entre 10 y 250 trabajadores, una antigüedad mínima de más de cinco años en el mercado. El universo de la población son 278 empresas de las cuales 199 corresponden a la categoría elegida para el estudio dando como resultado una muestra total de 118 empresas, la cual se determinó a través de la siguiente fórmula, para una población finita y un muestreo sin reemplazo:

$$
\begin{gathered}
\mathrm{n}=\frac{\mathrm{Z}^{2} \mathrm{Npq}}{\mathrm{e}^{2}(\mathrm{~N}-1)+\mathrm{Z}^{2} \mathrm{pq}} \\
\mathbf{n}=\mathbf{1 1 7 . 9 4 5 9} \approx \mathbf{1 1 8}
\end{gathered}
$$

\section{Instrumento de recolección de datos}

Para poder realizar la comparación de las respuestas y por ende su categorización y generalización, se utilizará la entrevista abierta apoyada por un cuestionario estructurado donde se analizan las estrategias de marketing dentro de las Pymes. Para estimar la confiabilidad del instrumento aplicado, se hizo uso del programa IBM SPSS (Paquete Estadístico para las Ciencias Sociales, por sus siglas en inglés), para obtener el Alfa de Cronbach en cada una de los items, obteniendo en lo general un coeficiente de .900; Posteriormente, con la finalidad de detectar errores de diseño y la factibilidad del instrumento, se llevó a cabo una prueba piloto con un mínimo de gerentes de empresas Pymes con las características de la muestra elegida, para de esta manera realizar los ajustes necesarios en las mismas y así aunada a la prueba de fiabilidad realizada a través del programa SPSS alcancen un elevado grado de confiabilidad. Las encuestas fueron aplicadas directamente a los gerentes de las empresas seleccionadas, que permita obtener 
Revista de Investigación

Académica sin Frontera

ISSN: 2007-8870

Núm. 31

\section{http://revistainvestigacionacademicasinfrontera.com}

Recibido el 12 de octubre de 2019. Dictaminado mediante arbitraje favorablemente 20 de diciembre de 2019.

información que sea verídica y confiable y, posteriormente capturada en el programa estadístico SPSS (Statistical Package for the Social Sciences), para su valoración e interpretación.

\section{Discusión de resultados}

Se presentan a continuación los resultados más relevantes de los avances de la investigación basados en el objetivo central de esta investigación:

Imagen 1; Uso de cambios estratégicos utilizados por las Pymes para enfrentar la competencia del mercado

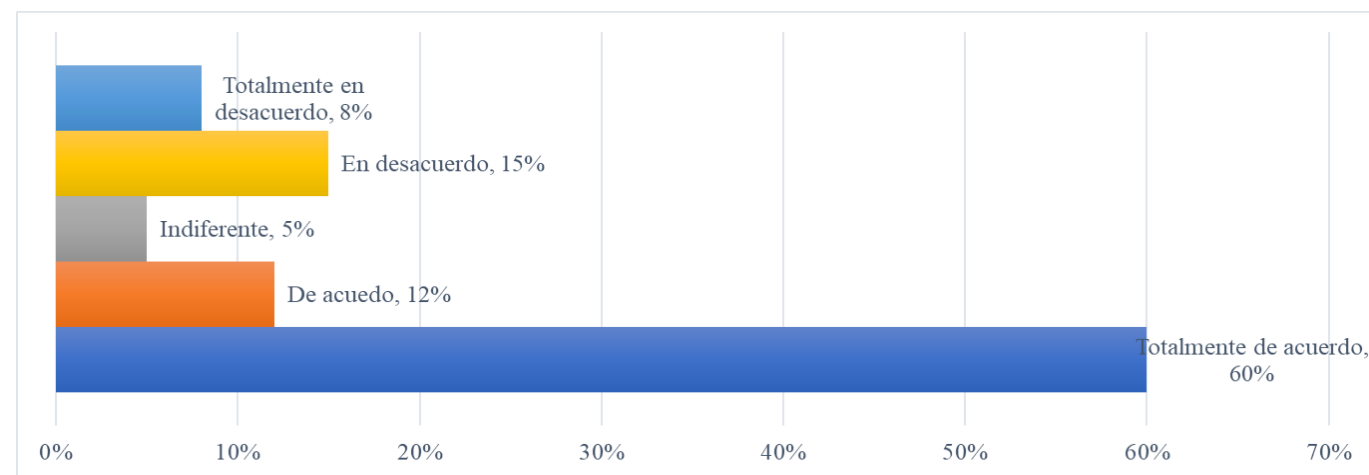

Fuente: Elaboración propia con base a los resultados estadísticos.

En la Imagen 1, se muestran los resultados donde el $60 \%$ de los gerentes opinan que estan totalmente de acuerdo que las Pymes, para enfrentar la competencia del mercado se plantea cambios estrategicos por los cuales no está preparada, el $15 \%$ está en desacuerdo, el $12 \%$ está de acuerdo, el $8 \%$ está totalmente en desacuerdo y el 5\% se muestra indiferente. Los anteriores resultados muestran que posiblemente el gerente Pyme está consciente de la competencia pero tambien al parecer desconoce la forma de crear estrategias para enfrentarla. 
Revista de Investigación

Académica sin Frontera

ISSN: 2007-8870

Núm. 31

\section{http://revistainvestigacionacademicasinfrontera.com}

Recibido el 12 de octubre de 2019. Dictaminado mediante arbitraje favorablemente 20 de diciembre de 2019.

Imagen 2; Principales estrategias de marketing utilizadas en las MIPymes

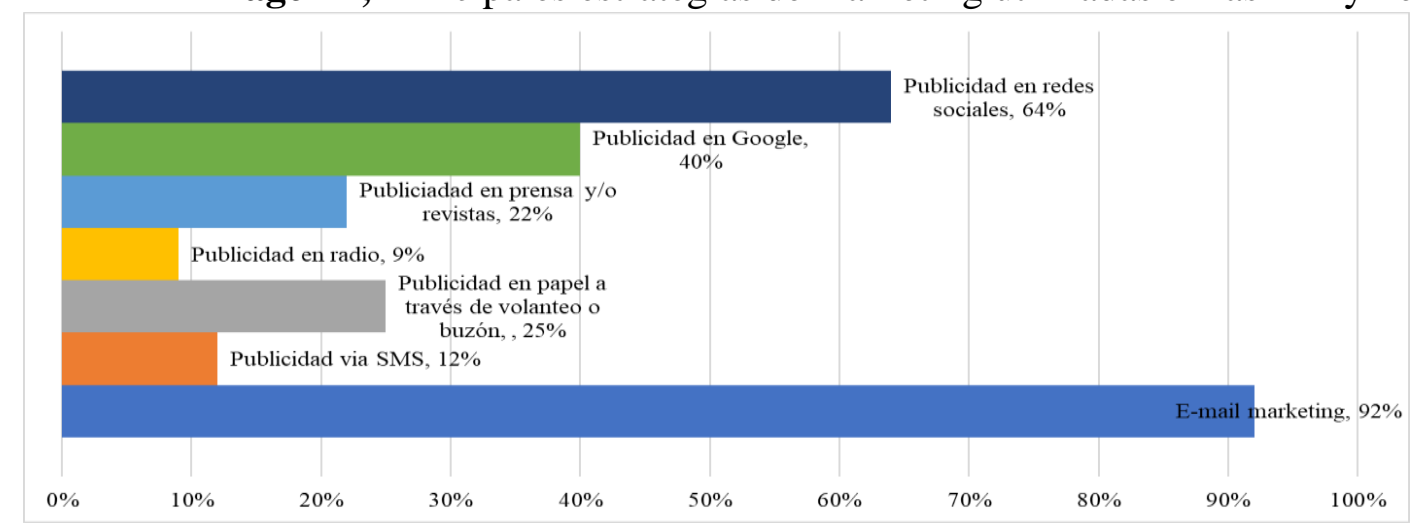

Fuente: Elaboración propia con base a los resultados estadísticos.

En la Imagen 2, se puede observar que el 92\% de las opiniones de los gerentes Pymes, utilizan el e-mail marketing para promocionar sus productos/servicios, el 64\% utiliza publicidad en redes sociales, el $40 \%$ publiciadad en google, el $25 \%$ utiliza publicidad en papel a través de volanteo o buzón, el $22 \%$ a través de prensa y/o revistas, el 12\% publicidad via SMS y el 9\% publicidad en radio. Los resultados hacen ver que el e-mail marketing, la publicidad en redes sociales y la publicidad en Google están prácticamente empatadas en la valoración de la empresas en cuanto a su eficacia, lo que hace suponer que, para estas empresas utilizan el marketing en la promoción de sus productos o servicios.

Imagen 3; La publicidad más valorada en las Pymes

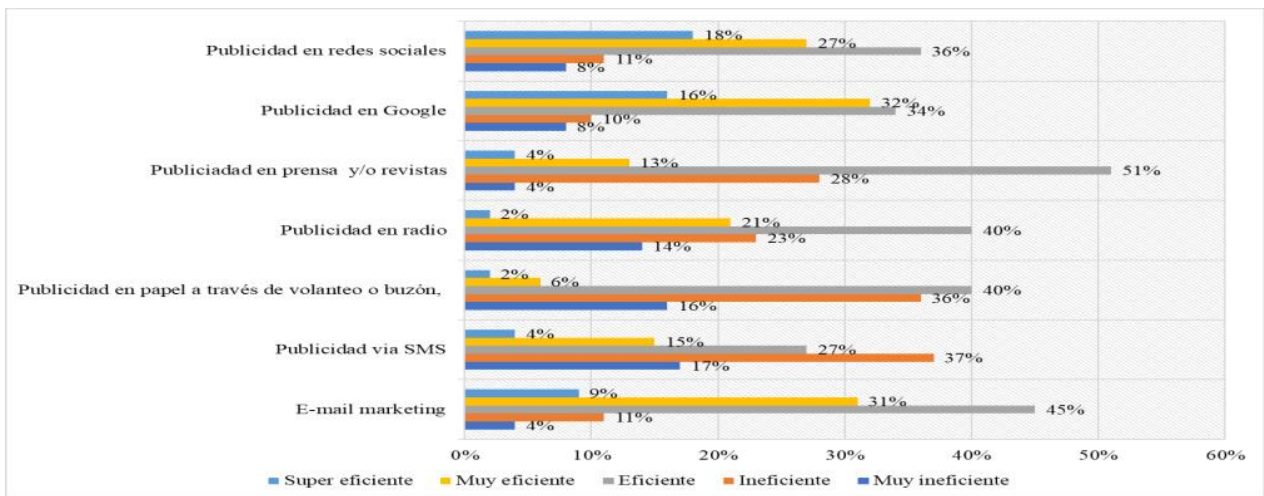

Fuente: Elaboración propia con base a los resultados estadísticos. 
Revista de Investigación

Año 12.

Académica sin Frontera

Núm. 31

ISSN: 2007-8870

\section{http://revistainvestigacionacademicasinfrontera.com}

Recibido el 12 de octubre de 2019. Dictaminado mediante arbitraje favorablemente 20 de diciembre de 2019.

En la Imagen 3, se muestran resultados donde el 51\% de los gerentes de las Pymes le da mas valor a la publicidad en prensa y/o revistas, el $45 \%$ al E-mail marketing, el $40 \%$ a la publicaida en radio y la publicidad en papel a través de volanteo o buzón, el 36\% le da valor a la publicidad en redes sociales, el 34\% a la publicidad en Google y el 27\% a la publicidad vía SMS. Los resultados hacen ver que entre las empresas que comercializan productos y servicios a otras empresas la estrategia de marketing más valorada es el e-mail marketing. El marketing en redes sociales adelanta a Google.

Imagen 4; Principal confianza del costo en marketing

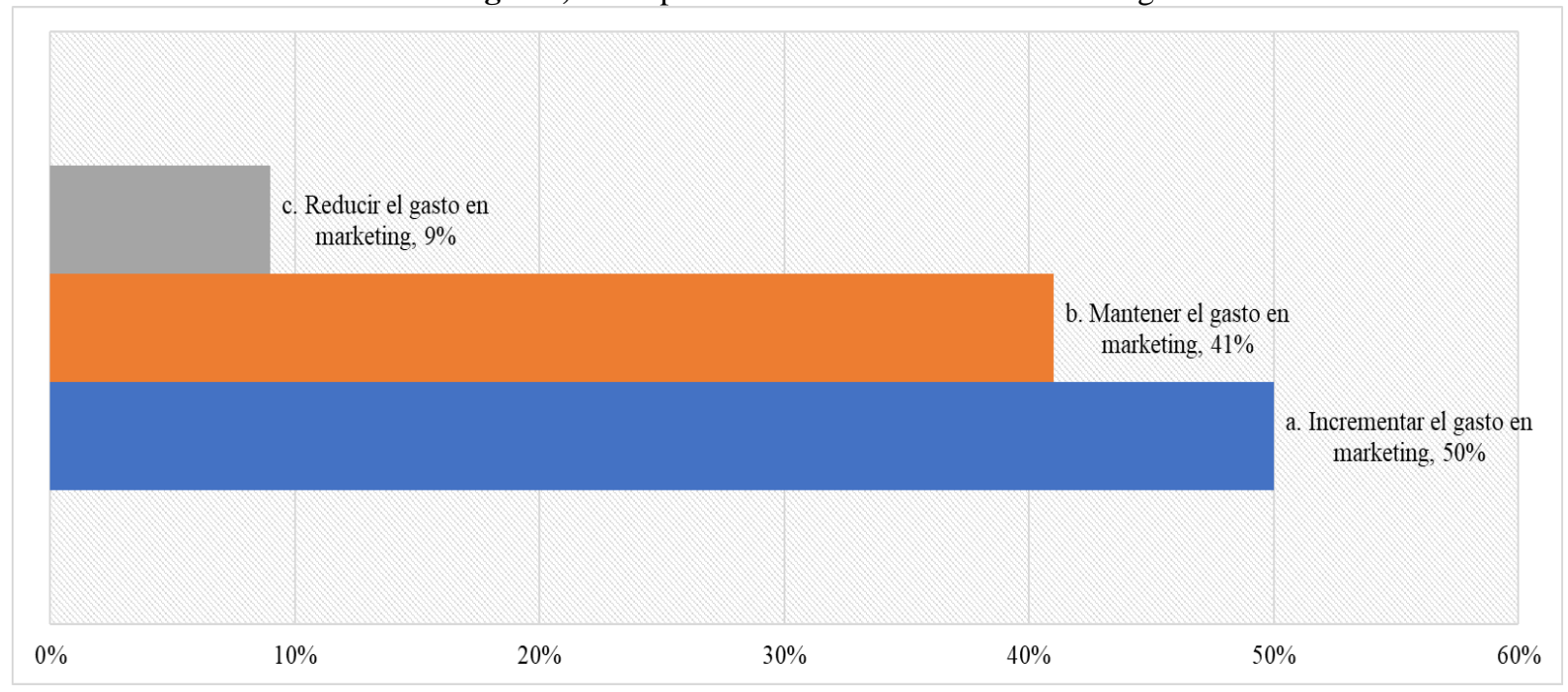

Fuente: Elaboración propia con base a los resultados estadísticos.

En la Imagen 4, se muestra resultados donde solo el 9\% de las opiniones de los gerentes de las Pymes esperan reducir su gasto de marketing en el siguiente año, contra el $91 \%$ que espera incrementar o mantener el mismo gasto de marketing. Los resultados anteriores, hacen suponer que las Pymes confían cada vez más en la recuperación del gasto invertido en marketing en el mismo año y en casi un $50 \%$ de los casos esperan incrementar su gasto en marketing en el siguiente año. 
Revista de Investigación

Académica sin Frontera

ISSN: 2007-8870

Núm. 31

\section{http://revistainvestigacionacademicasinfrontera.com}

Recibido el 12 de octubre de 2019. Dictaminado mediante arbitraje favorablemente 20 de diciembre de 2019.

Imagen 5; Porcentaje de gasto total en marketing es invertido a través de marketing en Internet y/o móviles

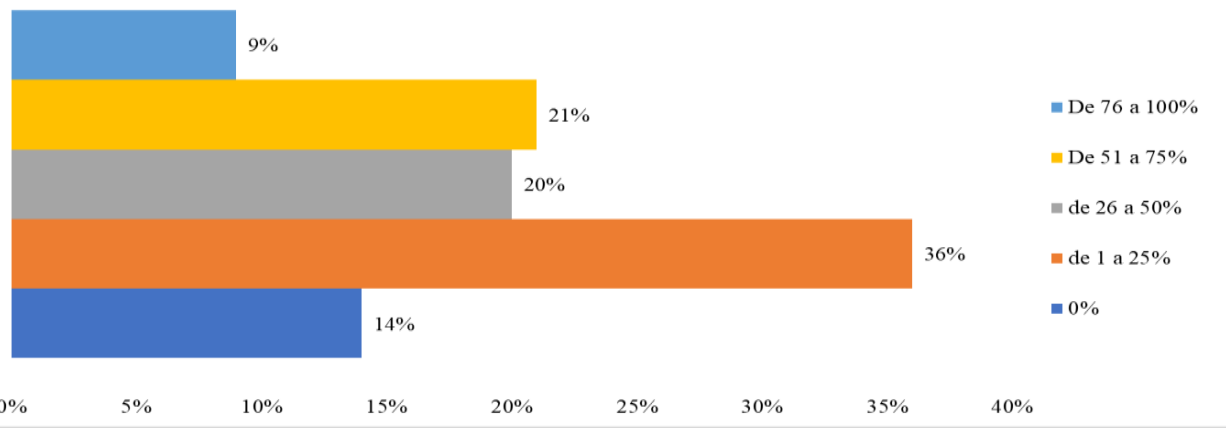

Fuente: Elaboración propia con base a los resultados estadísticos.

En la Imagen 5, se muestra los resultados donde de acuerdo a las opiniones del 36\% de las opiniones de los gerentes mencionan que el porcentaje de gasto total en marketing es invertido de 1 a $25 \%$ a través de marketing en Internet y/o móviles, el $21 \%$ opina que es de 51 a $75 \%$, el $20 \%$ opina que es de 26 a $50 \%$, para el $14 \%$ el porcentaje es un $0 \%$ y para el $9 \%$ de 76 a $100 \%$. Los resultados muestran que las Pymes invierten el 50\% de su gasto en el marketing digital, lo que hace suponer que su inversión es un poco conservadora, al parecer porque desconoce estrategias de marketing que le puedan servir o talvez no sabe usarlas.

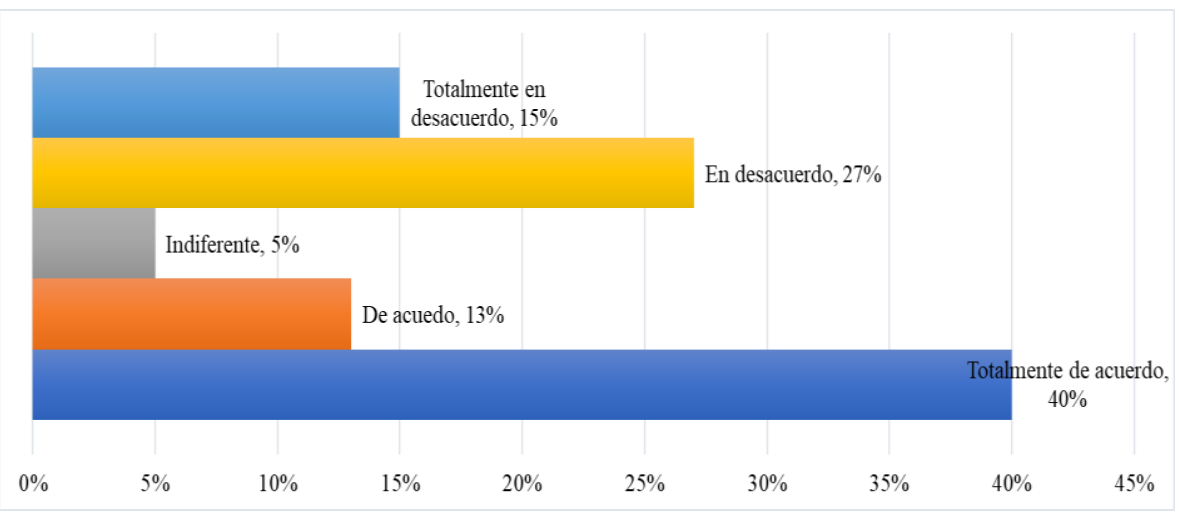

Fuente: Elaboración propia con base a los resultados estadísticos. 
Revista de Investigación

Académica sin Frontera

ISSN: 2007-8870

Núm. 31

\section{http://revistainvestigacionacademicasinfrontera.com}

Recibido el 12 de octubre de 2019. Dictaminado mediante arbitraje favorablemente 20 de diciembre de 2019.

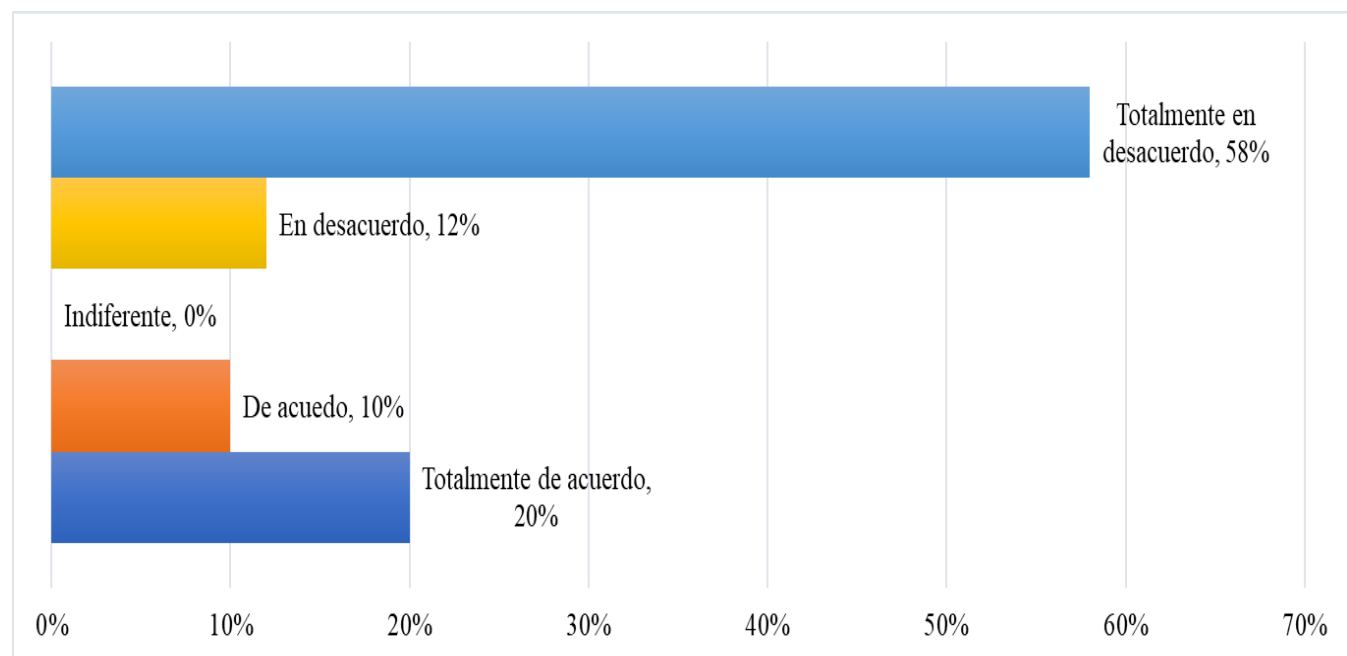

Fuente: Elaboración propia con base a los resultados estadísticos.

En la Imagen 6, se muestra que el 58\% de los gerentes opinan que están totalmente de acuerdo que el marketing utilizado en empresa es a través de asesoría externa, el $20 \%$ está totalmente de acuerdo, el $12 \%$ está en desacuerdo y el $10 \%$ de acuerdo. Los anteriores resultados muestran que al parecer las Pymes utilizan asesoría externa para realizar trabajo de marketing, al parecer estas empresas desconocen la forma de crear estrategias enfocadas a la aplicación de trabajo de marketing. 
"El saber de mis hijos hará mi grandeza"

Año 12.

Núm. 31
Revista de Investigación

Académica sin Frontera

ISSN: 2007-8870

\section{http://revistainvestigacionacademicasinfrontera.com}

Recibido el 12 de octubre de 2019. Dictaminado mediante arbitraje favorablemente 20 de diciembre de 2019.

Imagen 7. El trabajo de marketing utilizado en las Pymes parte de la opinión de los clientes y de un estudio de mercado previo con el objetivo final de crecer y ser competitivos

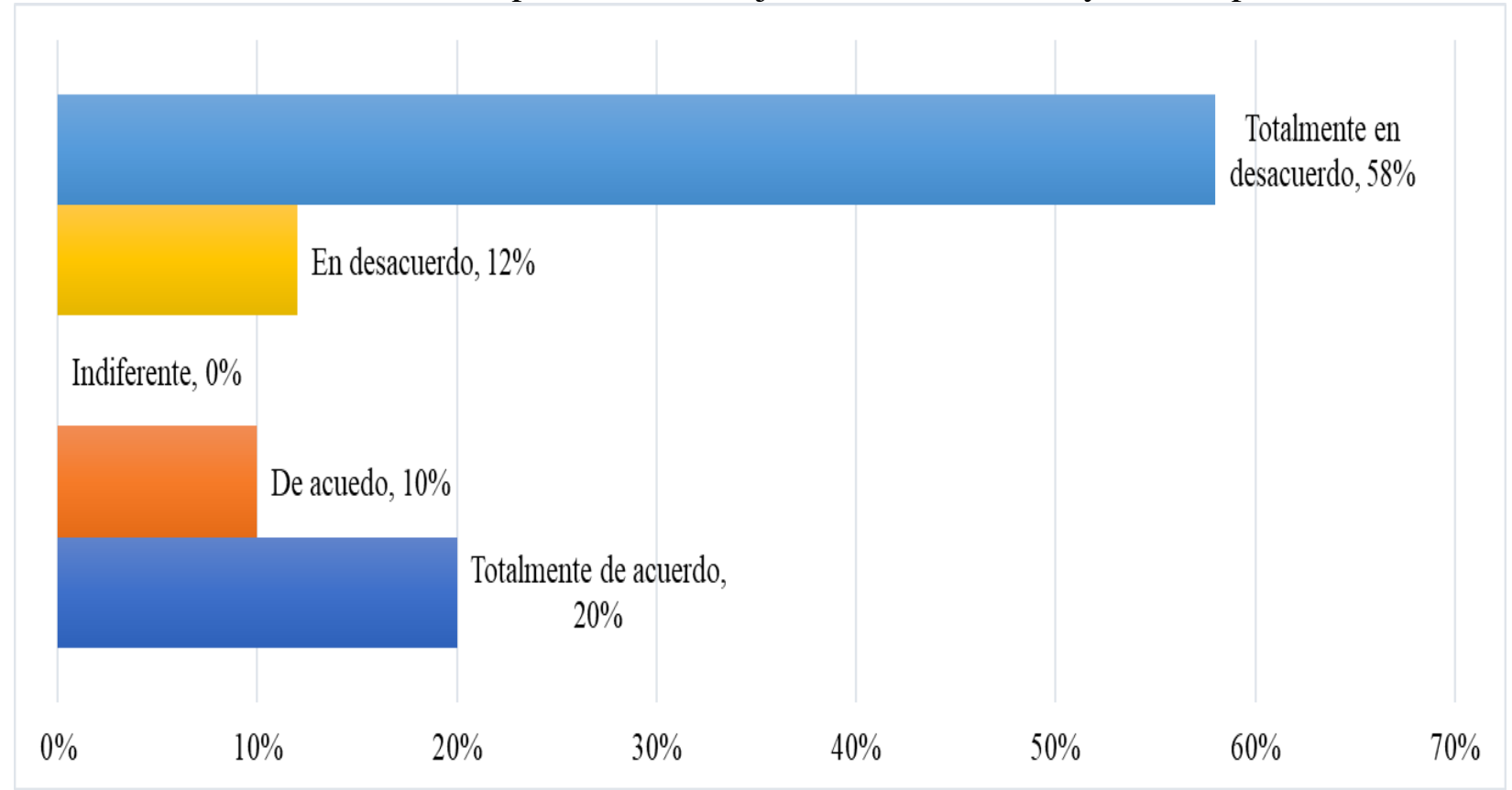

Fuente: Elaboración propia con base a los resultados estadísticos.

En la Imagen 7, se muestra que el $58 \%$ de los gerentes opinan que están totalmente en desacuerdo que el trabajo de marketing utilizado en las Pymes parte de la opinión de los clientes y de un estudio de mercado previo con el objetivo final de crecer y ser competitivos, el $20 \%$ opina que está totalmente de acuerdo, el 12\% opina que está en desacuerdo y el 10\% está de acuerdo. Los anteriores resultados muestran que al parecer el empresario de las Pymes 
"El saber de mis hijos hará mi grandeza"

Año 12.

Núm. 31
Revista de Investigación

Académica sin Frontera

ISSN: 2007-8870

\section{http://revistainvestigacionacademicasinfrontera.com}

Recibido el 12 de octubre de 2019. Dictaminado mediante arbitraje favorablemente 20 de diciembre de 2019.

desconoce la importancia que tiene en las estrategias de marketing, partir de las opiniones de los clientes y de un estudio de mercado previo.

\section{Conclusiones e implicaciones sociales y empresariales}

El marketing en cualquier empresa sea pequeña o grande va desde plantearse los objetivos a corto y largo plazo, hacer un análisis de su realidad interna y externa, delimitar bien al mercado y saber cómo es que queremos ser percibidos y con todo esto desarrollar estrategias de trabajo para poder conseguir todo aquello que nos estamos planteando. Los resultados aquí presentados, dan validez a los resultados obtenidos en la etapa exploratoria de la investigación y, permiten desde la opinión de los gerentes de las Pymes, hacer ver que dentro de las Pymes no se da importancia al marketing, y este tipo de empresas realizan una gestión de forma más tradicional, por experiencia personal o intuisión, sin darse cuenta que están haciendo marketing de la forma más rustica que existe. Sin embargo cada vez que se les habla de una estrategia de marketing piensan que esto es poco rentable y no lo ven solo desde el nivel monetario sino en la pérdida de tiempo que esto les puede ocasionar.

A modo de conclusión final y tomando en cuenta los planteamientos anteriores, se propone realizar dentro de las Pymes, estrategias de marketing a partir de un análisis de Fortalezas, Oportunidades, Debilidades y Amenazas (FODA), con el fín de generar un plan basado en estrategias acordes a las necesidades de los mercados globalizados que ayude a lograr ventaja competitiva. La realización de este proyecto se considera viable ya que Sonora, cuenta con un sector económico muy dinámico en sus diferentes sectores, tanto el industrial, el comercial y el de servicios que podría ayudar a través de estas propuestas a mejorar la gestión de sus negocios.

Este estudio presenta varias limitaciones que sugieren vías de investigación futuras. La percepción del marketing como herramienta de gestión empresarial de empresas de mediano y 
Revista de Investigación

Académica sin Frontera

ISSN: 2007-8870

\section{http://revistainvestigacionacademicasinfrontera.com}

Recibido el 12 de octubre de 2019. Dictaminado mediante arbitraje favorablemente 20 de diciembre de 2019.

mayor tamaño exitosas, es un resultado que debe analizarse para descartar la relatividad a su tamaño y éxito empresarial basado en su giro. Posteriormente, sería interesante profundizar en los efectos de la correlación entre otras variables que ayude en las estrategias de gestión empresarial para la mejora del trabajo que se realiza en materia de marketing y que le permitan lograr ventaja competitiva a la empresa. Asimismo, se propone trabajar en una nueva línea de investigación donde se realice el diseño de un plan de estrategias de marketing que ayude a estas empresas a lograr una gestión empresarial acorde a las necesidades de estas empresas para que puedan enfrentar la competitividad actual.

\section{Bibliografía}

Agius, A. (2015). Haz marketing como los grandes. Entrepeneur, 42-46.

Alarcón Chávez, C.R. y Granda García, M.I. (2018). El marketing y la fidelización empresarial como apuesta estratégica para pymes en Ecuador. Revista Científica Dominio de la Ciencia. 4 (1), 131-140.

Carson, D. and Gilmore, A. (2000), Marketing at the interface: Not 'what' but 'how'. Journal of Marketing Theory and Practice, 8(2), 1-7.

Coviello, N. Brodie, R. Munro H. (2000) An Investigation of marketing practice by firm size. Journal of Business Venturing 15, 523-545.

Gilmore, A. Carson D. Grant, J. (2001). SME Marketing in practice. Marketing Intelligence \& Planning. 19/1. pp 6-11.

Góngora, G., y Madrid, A. (2010). El apoyo a la innovación de la pyme en México. Un estudio exploratorio, Investigación y Ciencia, vol. 18, núm. 47, pp. 21-30. 
Revista de Investigación

Académica sin Frontera ISSN: 2007-8870

\section{http://revistainvestigacionacademicasinfrontera.com}

Recibido el 12 de octubre de 2019. Dictaminado mediante arbitraje favorablemente 20 de diciembre de 2019.

Gruber, M. (2004). Marketing in new ventures: Theory and empirical evidence. Schmalenbach Business Review, 56, 164-199.

Hills, G. Hultman, M. y Morgan, M. (2008). The evolution and development of Entrepreneurial Marketing. Journal of Small Business Management. 99-112

Instituto Nacional de Estadística y Geográfica. (febrero de 2015). Esperanza de vida de los negocios. Recuperado el 12 de marzo de 2019, de www.inegi.org: http://www.inegi.org.mx/saladeprensa/boletines/2015/especiales/especiales2015_02_38.pdf

Jaworski Bernard, J. y Kohli Ajay, K. (1993), Market Orientation: Antecedents and Consequences, Journal of Marketing, Vol. 57, 53-70.

Kotler, P. y Amstrong, F. (2010). Dirección de Marketing. Prentice Hall, 12, 6.

Lamb, Ch., Hair, J., y McDaniel, C. (2011). Marketing. México: Cengage Learning.

Marcati, A., Guido, G. and Peluso, A. (2008). What is marketing for SME entrepreneurs? The need to market the marketing approach. Congress International "Marketing Trends", Venice, Italy, 17-19 January.

Martin, D. (2009) The entrepreneurial marketing mix. Qualitative Market Research: An International Journal, 12, (4), 391-403.

McCartan-Quinn, D. y Carson, D. (2003). Issues wich Impact upon Marketing in the Small Firm. Small Business Economics 21, 201-203.

Morrish, S. y Deacon, J. (2009). Entrepreneurial Marketing: A comparative case study of 42Below Vodka and Penderyn Whisky. Sussie Morrish: The University Of Auckland, Auckland, New Zealand. AGSE 2009, 887-901.

O'Dwyer, M., Gilmore, A., Carson, D. (2009) Innovative marketing in SMEs. European Journal of Marketing. 43(1/2), 46-61. 
"El saber de mis hijos hará mi grandeza"

Año 12.

Núm. 31
Revista de Investigación

Académica sin Frontera

ISSN: 2007-8870

\section{http://revistainvestigacionacademicasinfrontera.com}

Recibido el 12 de octubre de 2019. Dictaminado mediante arbitraje favorablemente 20 de diciembre de 2019.

R. Paredes, M. (2011). El proceso del marketing en las pequeñas empresas. ECO revista académica (6), 56. Recuperado el 31 de agosto de 2019, de http://biblio3.url.edu.gt/Revistas/ECO/ECO-2011-06.pdf\#page=56

Reijonen, H. (2010). Do all SMEs practise same kind of marketing?. Journal of Small Business and Enterprise Development, 17(2), 279-293.

Reijonen, H. (2010). Do all SMEs practise same kind of marketing?. Journal of Small Business and Enterprise Development, 17, 2, 279-293.

Stokes, D. (2000). Putting Entrepreneurship into Marketing: The Processes of Entrepreneurial Marketing. Journal of Research in Marketing and Entrepreneurship, 2,1,1-16.

Villalobos Nivón, J. (mayo de 2015). Conquista Programada. Entrepeneur, 25-28.

Zontanos, G. Anderson, A. (2004). Relationships, marketing and small business: an exploration of links in theory and practice. Qualitative Market Research: An International Journal. 7(3), 228-23. 


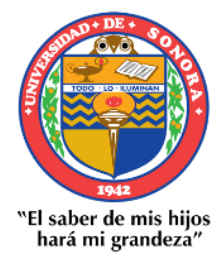

Año 12.

Núm. 31
( Julio - Diciembre 2019 )

Revista de Investigación

Académica sin Frontera ISSN: 2007-8870

\section{http://revistainvestigacionacademicasinfrontera.com}

Recibido el 12 de octubre de 2019. Dictaminado mediante arbitraje favorablemente 20 de diciembre de 2019.

\section{Directorio Institucional}

\section{Dr. Enrique Fernando Velázquez Contreras}

Rector

Dra. Guadalupe García de León Peñuñuri

Secretario General Académico

Dra. Rosa Mará Montesinos Cisneros

Secretaria General Administrativa

Dra. Ramón Enrique Robles Zepeda

Director de Investigación y Posgrado

Dr. Rodolfo Basurto Álvarez

Director de Vinculación y Difusión

Dra. Adriana Leticia Navarro Verdugo

Vicerrectora de la Unidad Regional Sur

Dr. Ernesto Clark Valenzuela

Director de la División de Ciencias Económicas y Sociales

Dr. Francisco Espinoza Morales

Secretario de la División de Ciencias Económico y Sociales

Mtra. María Guadalupe Alvarado Ibarra

Jefe del Departamento de Ciencias Económico Administrativas

Dra. Lidia Amalia Zallas Esquer

Jefe de Departamento de Ciencias Sociales 


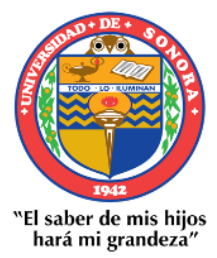

Año 12.

Núm. 31
( Julio - Diciembre 2019 )

Revista de Investigación

Académica sin Frontera ISSN: 2007-8870

\section{http://revistainvestigacionacademicasinfrontera.com}

Recibido el 12 de octubre de 2019. Dictaminado mediante arbitraje favorablemente 20 de diciembre de 2019.

\section{Directorio}

\section{Editor Responsable}

Dr. Francisco Espinoza Morales

Directora

Dra. Leticia María González Velásquez

Subdirector

Dr. Javier Carreón Guillen

Editor Científico

Dr. Cruz García Lirios

Master Gráfico

M.T.I. Francisco Alan Espinoza Zallas 
"El saber de mis hijos hará mi grandeza"

Año 12.

Núm. 31
Revista de Investigación

Académica sin Frontera ISSN: 2007-8870

http://revistainvestigacionacademicasinfrontera.com

Recibido el 12 de octubre de 2019. Dictaminado mediante arbitraje favorablemente 20 de diciembre de 2019.

\section{Comité editorial}

Dra. Angélica María Rascón Larios

Universidad de Sonora. México

Dra. María del Rosario Molina González

Universidad de Sonora

Dra. Francisca Elena Rochin Wong

Universidad de Sonora. México

Dra. Lidia Amalia Zallas Esquer

Universidad de Sonora. México

Dra. Beatriz Llamas Arechiga

Universidad de Sonora. México

Dr. Rogelio Barba Álvarez

Universidad de Guadalajara. México

Dra. Rosa María Rincón Ornelas

Universidad de Sonora. México

Dr. Juan Flores Preciado

Universidad de Colima. México

Dr. Amado Olivares Leal. Universidad de Sonora

Universidad de Sonora. México

Dr. Guillermo Velásquez Valadez.

Instituto Politécnico Nacional (IPN) México 


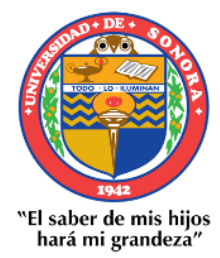

Año 12.

Núm. 31
( Julio - Diciembre 2019 )

Revista de Investigación

Académica sin Frontera ISSN: 2007-8870

\section{http://revistainvestigacionacademicasinfrontera.com}

Recibido el 12 de octubre de 2019. Dictaminado mediante arbitraje favorablemente 20 de diciembre de 2019.

Dr. Hugo Nefstalí Padilla Torres.

Universidad Estatal de Sonora. México

Dr. Luis Ramón Moreno Moreno.

Universidad Autónoma de Baja California. México

Dr. Miguel Ángel Vázquez Ruiz.

Universidad de Sonora. México

Dra. Lorena Vélez García.

Universidad Autónoma de Baja California. México

Dra. Pabla Peralta Miranda.

Universidad Simón Bolívar, Barranquilla, Colombia

Mtro. Roberto Espíritu Olmos

Universidad de Colima (FCA Tecomán) Colima

Dr. Héctor Priego Huertas.

Universidad de Colima (FCA Tecomán) Colima

Mtra. María Guadalupe Alvarado Ibarra.

Universidad de Sonora. México.

Revisores de Textos en Inglés

Mtra. Cecilia Guadalupe Martínez Solano

Dra. María del Socorro Vega Mosqueda 


\section{http://revistainvestigacionacademicasinfrontera.com}

Recibido el 12 de octubre de 2019. Dictaminado mediante arbitraje favorablemente 20 de diciembre de 2019.

\section{Comité científico}

Dr. Rosendo Martínez Jiménez. Universidad Autónoma Benito Juárez de Oaxaca.

Dr. Hugo Neftalí Padilla. Universidad Estatal de Sonora

Dra. María Teresa Gaxiola Sánchez. Universidad de Sonora.

Dr. José Cesar Kaplan. Universidad estatal de Sonora.

Dr. Alfredo Islas Rodríguez. Universidad de Sonora

Frecuencia de publicación: semestral / 2 números por año.

Revista de Investigación Académica sin Frontera (RIASF) con (ISSN: 2007-8870) es un interlocutor internacional de acceso abierto revisado diario en línea en el ámbito del de las Ciencias Económicas Administrativas y Sociales. Su objetivo principal es dar a los trabajos de investigación de calidad. Cubre todas las sub-campos de los campos anteriormente mencionados. Proporciona la plataforma a académicos, estudiantes y profesionales. Sólo pública trabajos de investigación y artículos de revisión inicial. Documento presentado debe cumplir con algunos criterios como, debe ser original, inédita y no estén sometidos a ninguna otra revista.

RIASF es una revista arbitrada / Revisión por pares International. Publicamos documentos sobre una variedad de temas, contextos y estrategias de análisis que examinan la relación entre la rápida evolución para la Sociedad y la tecnología del conocimiento.

REVISTA DE INVESTIGACIÓN ACADÉMICA SIN FRONTERA, Año 12, No. 31, julio - diciembre 2019, es una publicación semestral de investigación científica, editada por la Universidad de Sonora, a través de las División de Ciencias Económicas y Sociales, de la unidad regional Sur, Blvd. Lázaro Cárdenas No. 100, Col. Francisco Villa, Navojoa, Sonora, Sonora, México, C.P. 85880. Tel. (642) 425- 99-54.

http://www.revistainvestigacionacademicasinfrontera.com/, fespinoz@navojoa.uson.mx.

Editor responsable: Francisco Espinoza Morales. Reserva de Derechos al Uso Exclusivo: 04-2013121811323700-203 e ISSN: 2007-8870, ambos otorgados por el Instituto Nacional de Derecho de Autor. Inscrita en el Directorio de LATINDEX, con Núm. De folio 20014, folio único 14590. Responsable de la última actualización de este Número, Unidad Informática de la Universidad de Sonora, fecha de la última modificación, 31 de diciembre 2019. Las opiniones expresadas por los autores no necesariamente reflejan la postura del editor de la publicación. Se autoriza la reproducción total o parcial de los contenidos e imágenes en la presente publicación siempre y cuando se cuente con la autorización del editor y se cite plenamente la fuente. 